\title{
Topology of pocket formation in turbulent premixed flames
}

\author{
S. Trivedi ${ }^{\mathrm{a}, *}$, R. Griffiths ${ }^{\mathrm{a}}$, H. Kolla ${ }^{\mathrm{b}}$, J.H. Chen ${ }^{\mathrm{b}}$, R.S. Cant ${ }^{\mathrm{a}}$ \\ ${ }^{a}$ Department of Engineering, University of Cambridge, Cambridge CB2 1PZ, United \\ Kingdom \\ ${ }^{b}$ Combustion Research Facility, Sandia National Laboratories, Livermore CA 94550, \\ United States
}

\begin{abstract}
In turbulent premixed flame propagation, the formation of isolated pockets of reactants or products is associated with flame pinch-off events which cause rapid changes in the flame surface area. Previous topological analysis of these phenomena has been carried out based on Morse theory and Direct Numerical Simulation (DNS) in two spatial dimensions. The present work extends the topological analysis to three dimensions with emphasis on the formation and subsequent burnout of reactant pockets. Singular behaviour observed previously for terms of the Surface Density Function (SDF) transport equation in the two-dimensional case is shown to occur also in three dimensions. Further singular behaviour is observed in the displacement speed close to reactant pocket burnout. The theory is compared against DNS data from hydrogen-air flames and good agreement is obtained.
\end{abstract}

Keywords:

Direct Numerical Simulation, turbulent premixed flames, flame surface

\footnotetext{
*Corresponding author:

Email address: st634@cam.ac.uk (S. Trivedi)
} 
topology, pocket formation

\section{Introduction}

Premixed turbulent flames often consist of a thin and highly wrinkled layer of reacting gas forming an interface between extended regions of reactant and products. The local geometry of the interface has a profound influence on the propagation of the flame and hence on the overall turbulent burning rate, which is a quantity of great interest in technological applications, for example in spark-ignition internal combustion engines and gas turbines. The statistical geometry of the flame surface is commonly modelled using the Flame Surface Density (FSD) [1] and G-equation [2] approaches, both of which require fundamental information about the local evolution of the interface. Previous work [3-5] has made use of topological concepts to determine the range of possible geometrical configurations of the flame surface and most importantly to provide estimates of their relative frequency of occurrence.

Among the most important events in terms of its effect on the total flame surface area is flame pinch-off. This occurs kinematically when two adjacent flame surfaces collide, leading to the occurrence of a critical point and the formation of a pocket. This process has been analysed in detail for the two-dimensional case of head-on collision and the formation of a pocket of reactants $[4,6]$. It was shown that a singularity occurs at the critical point, leading to rapid recession of the interface during pocket formation.

More recently, topological analysis [5] of three dimensional flame surfaces has identified all of the possible surface topologies that can occur in the vicin- 
ity of a critical point at which the scalar gradient is equal to zero. The full range of topologies is shown in Fig.1. Moreover, the analysis indicated that there are four principal configurations which occur most frequently on the flame surface. These are 1) reactant pockets and 2) product pockets, together with 3) tunnel closure and 4) tunnel formation. A reactant pocket is topologically spherical and propagates inwards (see Fig.1, bottom right corner), while a product pocket differs by propagating outwards (top left). Tunnel closure involves inward propagation of a topologically cylindrical flame (bottom row, third from right), while tunnel formation involves outward propagation (top row, third from left).

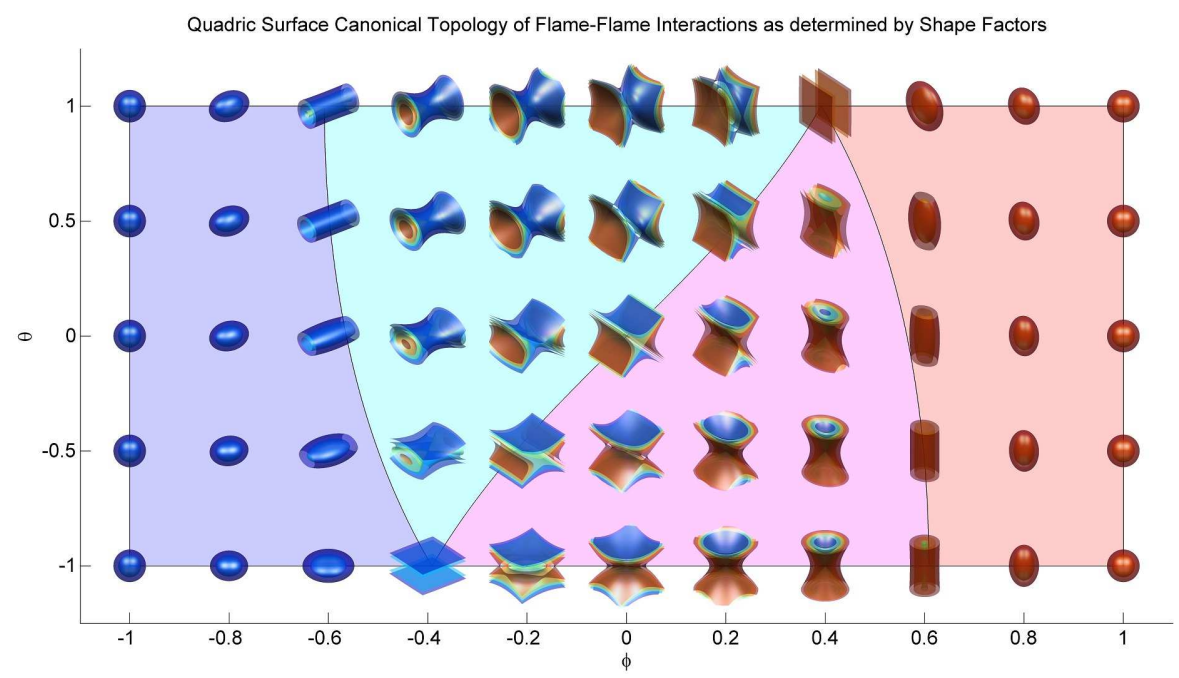

Figure 1: All possible flame surface topologies. From [5]. The axes represent shape factors derived from scaled eigenvalues of the Hessian of the reaction progress variable.

It is evident on physical grounds that tunnel closure may lead to pinchoff and the formation of a reactant pocket. Hence this configuration offers the opportunity to extend the previous two-dimensional analysis to three 
dimensions, and to gain some insight into the kinematics of the pinch-off event and its effect on flame surface area. The other configurations of interest are also amenable to similar treatment.

The purpose of the present paper is to analyse the four principal flame configurations previously identified in three dimensions by Griffiths et al [5], and to validate the findings of the theoretical analysis by using DNS data for the particular case of reactant pocket formation. The previous two-

dimensional topological approach of Kollmann and Chen [4] is extended using a similar theoretical basis [7] to the much richer topology accessible in three dimensions. It is demonstrated that the theory remains valid in three dimensions despite the additional topological complexity and indeed allows for similar physical conclusions. The results confirm that singular behaviour occurs during three-dimensional pinch-off events, and during the final closure of reactant pockets.

\section{Mathematical background}

A reaction progress variable $c$ may be defined (e.g.) in terms of normalised product species mass fraction such that $c=0$ in fresh reactants and $c=1$ in fully-burned products. A balance equation for $c$ may be written as

$$
\rho \frac{\partial c}{\partial t}+\rho u_{k} \frac{\partial c}{\partial x_{k}}=w+\frac{\partial}{\partial x_{k}}\left(\rho D \frac{\partial c}{\partial x_{k}}\right)
$$

where $\rho$ is the density, $u_{i}$ is the velocity, $D$ is the diffusivity of $c$ and $w$ is the chemical production rate of $c$.

The surface density function $\sigma$ is defined as the magnitude of the gradient of $c$, i.e. $\sigma=|\nabla c|$, while the normal vector to an isosurface of $c$ is defined 
as $\underline{n}=-\nabla c /|\nabla c|$, pointing down the gradient, i.e. in the direction of flame propagation. The balance equation for $\sigma$ may be stated [4] as

$$
\frac{\partial \sigma}{\partial t}+u_{k} \frac{\partial \sigma}{\partial x_{k}}=-n_{i} n_{k} \sigma \frac{\partial u_{i}}{\partial x_{k}}+n_{k} \frac{\partial}{\partial x_{k}}(D \kappa \sigma)+n_{k} \frac{\partial}{\partial x_{k}}\left[\frac{w}{\rho}+\frac{n_{j}}{\rho} \frac{\partial}{\partial x_{j}}(\rho D \sigma)\right]
$$

where $\kappa=\partial n_{k} / \partial x_{k}$ is (twice) the curvature of the flame surface. The three terms on the right-hand side of this equation are named respectively as the strain rate term, the dissipation term and the kinematic restoration term [4]. Note that the kinematic restoration term itself contains two contributions arising from reaction and from normal restoration.

Considering only the local topology, it is possible to represent each of the four principal configurations (reactant pocket RP, product pocket PP, tunnel closure TC and tunnel formation TF) in scaled local coordinates $x, y$ and $z$ for small deviations from the critical point. The expressions are:

$$
\begin{aligned}
& (\mathrm{RP}) \quad c(x, y, z)=c_{0}+\frac{1}{2} x^{2}+\frac{1}{2} y^{2}+\frac{1}{2} z^{2} \\
& (\mathrm{PP}) \quad c(x, y, z)=c_{0}-\frac{1}{2} x^{2}-\frac{1}{2} y^{2}-\frac{1}{2} z^{2} \\
& (\mathrm{TC}) \quad c(x, y, z)=c_{0}-\frac{1}{2} x^{2}+\frac{1}{2} y^{2}+\frac{1}{2} z^{2} \\
& (\mathrm{TF}) \quad c(x, y, z)=c_{0}+\frac{1}{2} x^{2}-\frac{1}{2} y^{2}-\frac{1}{2} z^{2}
\end{aligned}
$$

where $c_{0}$ is the value of the progress variable at the critical point. A reactant pocket corresponds to a local minimum, with a low value of progress variable in the centre with higher values all around. For this configuration the normal vector is $\underline{n}=(-x / r,-y / r,-z / r)^{T}$ and the curvature $\kappa=-2 / r$, where the radius $r=\left(x^{2}+y^{2}+z^{2}\right)^{1 / 2}$. For a product pocket the same quantities are given by $\underline{n}=(x / r, y / r, z / r)^{T}$ and $\kappa=2 / r$. 
A tunnel closure corresponds to a local saddle point with a maximum on the central axis of the tunnel (taken here as the $x$ direction) combined with a minimum in the other two directions. The normal vector is $\underline{n}=$ $(x / r,-y / r,-z / r)^{T}$ and the curvature is $\kappa=-2 x^{2} / r^{3}$. For tunnel formation the normal vector is $\underline{n}=(-x / r, y / r, z / r)^{T}$ and the curvature is $\kappa=2 x^{2} / r^{3}$. For all four configurations the SDF is given by $\sigma=r$.

For the tunnel closure configuration, it is interesting to examine the behaviour of the last two terms in the SDF balance equation (2). The dissipation term can be expanded as

$$
n_{k} \frac{\partial}{\partial x_{k}}(D \kappa \sigma)=n_{k}(\kappa \sigma) \frac{\partial D}{\partial x_{k}}+n_{k} D \frac{\partial(\kappa \sigma)}{\partial x_{k}}
$$

and the two terms in the right-hand side may be evaluated close to the critical point as

$$
\begin{aligned}
n_{k}(\kappa \sigma) \frac{\partial D}{\partial x_{k}} & =-\frac{2 x^{2}}{r^{3}}\left(x \frac{\partial D}{\partial x}+y \frac{\partial D}{\partial y}+z \frac{\partial D}{\partial z}\right) \\
n_{k} D \frac{\partial(\kappa \sigma)}{\partial x_{k}} & =-8 D\left(\frac{x^{2} y^{2}+x^{2} z^{2}}{r^{5}}\right)
\end{aligned}
$$

In the limit of small $r$, as the critical point is approached, the first term (eq. 8) remains bounded but the second term (eq. 9) becomes singular, behaving like $1 / r$ as $r \rightarrow 0$. This result is consistent with the previous two-dimensional analysis [4].

A similar examination may be carried out for the kinematic restoration term. It may be shown readily that the reactive restoration term remains bounded close to the critical point for all configurations. The normal restoration term may be decomposed into five terms according to $n_{k} \frac{\partial}{\partial x_{k}}\left[\frac{n_{j}}{\rho} \frac{\partial}{\partial x_{j}}(\rho D \sigma)\right]=D n_{k} \frac{\partial n_{j}}{\partial x_{k}} \frac{\partial \sigma}{\partial x_{j}}+D n_{k} n_{j} \frac{\partial^{2} \sigma}{\partial x_{k} \partial x_{j}}+n_{k} \frac{\partial n_{j}}{\partial x_{k}} \frac{\sigma}{\rho} \frac{\partial}{\partial x_{j}}(\rho D \sigma)$ 


$$
+n_{k} n_{j} \frac{\partial D}{\partial x_{k}} \frac{\partial \sigma}{\partial x_{k}}+n_{k} n_{j} \frac{\partial}{\partial x_{k}}\left[\frac{\sigma}{\rho} \frac{\partial}{\partial x_{j}}(\rho D)\right]
$$

All of these terms may be evaluated using the expressions for $n_{i}$ and $\sigma$ as stated above. For the tunnel closure configuration, the last three terms remain bounded. The first two terms may be evaluated together as

$$
D n_{k} \frac{\partial n_{j}}{\partial x_{k}} \frac{\partial \sigma}{\partial x_{j}}+D n_{k} n_{j} \frac{\partial^{2} \sigma}{\partial x_{k} \partial x_{j}}=4 D\left(\frac{x^{2} y^{2}+x^{2} z^{2}}{r^{5}}\right)+4 D\left(\frac{x^{2} y^{2}+x^{2} z^{2}}{r^{5}}\right)
$$

In the limit of small $r$, these terms become singular, again behaving like $1 / r$ as $r \rightarrow 0$. Moreover, together they cancel exactly with the singular term arising from the dissipation (see eq. 9). Hence the balance equation for $\sigma$ remains bounded, and indeed $\sigma \rightarrow 0$ in the limit. Again, these findings are consistent with the previous two-dimensional analysis [4]. Similar singular behaviour may be observed in the tunnel formation case.

By contrast, no singularities arise in the terms of the $\sigma$ equation for either of the pocket configurations even for the final burnout of a reactant pocket. Here, further insight can be gained by considering the displacement speed $S_{d}$ defined with reference to the right-hand side of the $c$-equation (1) according to

$$
\rho S_{d} \sigma=w+\frac{\partial}{\partial x_{k}}\left(\rho D \frac{\partial c}{\partial x_{k}}\right)
$$

The displacement speed may be split into contributions arising from reaction, normal diffusion and curvature according to

$$
S_{r}=\frac{w}{\rho \sigma} ; \quad S_{n}=-\frac{n_{k}}{\rho \sigma} \frac{\partial}{\partial x_{k}}(\rho D \sigma) ; \quad S_{t}=-D \kappa
$$

The contribution due to normal diffusion may be further decomposed as

$$
S_{n}=-\frac{n_{k}}{\rho} \frac{\partial}{\partial x_{k}}(\rho D)-\frac{n_{k} D}{\sigma} \frac{\partial \sigma}{\partial x_{k}}
$$


Evaluation for the tunnel closure configuration indicates that the first term remains bounded. The second term may be combined with the curvature term to yield

$$
S_{n}+S_{t}=-D\left[\frac{x^{2}}{r^{3}}-\frac{y^{2}}{r^{3}}-\frac{z^{2}}{r^{3}}\right]+2 D \frac{x^{2}}{r^{3}}=\frac{D}{r}
$$

which becomes singular as $r \rightarrow 0$ provided that $D$ remains non-zero in the limit. Note that the reactive term also becomes singular provided that $w$ remains non-zero in the limit. Hence the displacement speed is unbounded during tunnel closure. Similar behaviour is evident in the displacement speed during reactant pocket burnout. It is important to note that the quantity $S_{d} \sigma$ remains bounded in all configurations, and hence both the progress variable equation and the SDF equation remain well-behaved.

\section{DNS dataset}

Here the analysis is carried out using the DNS data of Hawkes et al. [8] generated for two initially planar lean $\mathrm{H}_{2}$-air flames propagating towards each other in a high intensity shear-driven turbulent flow. The equivalence ratio was 0.7 and the mixture was preheated to $700 \mathrm{~K}$. A 9-species 21-step reaction mechanism was employed [9]. The dataset was produced using the DNS code S3D [10] which employs an 8th order spatial finitedifference scheme and 4th order explicit Runge-Kutta time-stepping. The computational domain was a cuboid of physical size $16 H \times 20 H \times 12 H$, discretised using $2400 \times 1600 \times 1800$ mesh points. The value of $H$ was $5.4 \mathrm{~mm}$, and the mesh spacing was $36 \mu \mathrm{m}$. The integral length scale was $H / 3$. Boundary conditions were set to be periodic in the streamwise and spanwise directions, 
with outflow in the direction perpendicular to the flames. The full dataset involves two different Damköhler numbers denoted as Da- (Da=0.13) and $\mathrm{Da}+(\mathrm{Da}=0.54)$. Only the Da+ data is used for the present study, and a small subsection of the entire dataset was enough to find sufficient numbers of critical point for analysis. The temperature field for this subset is shown in Fig.2.

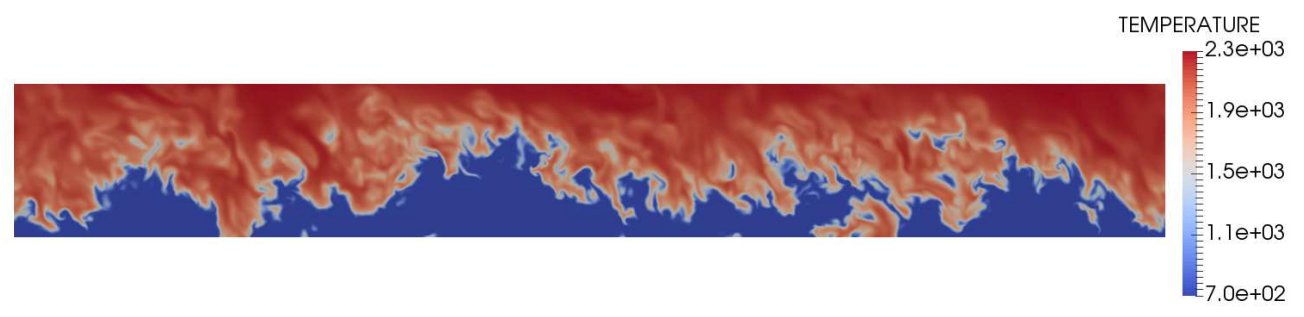

Figure 2: Subset of Da+ dataset from Hawkes et al. [8]

It can be seen that the flame is highly wrinkled, but it should be noted that the flame structure remains intact, i.e., the reactants and products are separated by a continuous flame surface. No broken reaction zones were observed.

\section{Results and discussion}

For the purposes of analysis, a reaction progress variable is defined based on $\mathrm{O}_{2}$ mass fraction according to

$$
c=\frac{Y_{\mathrm{O}_{2}, R}-Y_{\mathrm{O}_{2}}}{Y_{\mathrm{O}_{2}, R}-Y_{\mathrm{O}_{2}, P}}
$$

The value of the progress variable thus defined rises monotonically from zero in the reactants to unity in the products. The progress variable gradient is 
used to evaluate quantities such as the flame normal $\underline{n}$, curvature $\kappa$ and SDF $\sigma$. Hence it is possible to evaluate the terms of the SDF balance equation, and especially the dissipation and kinematic restoration terms in the vicinity of each critical point (see eqs. 9 and 11). Similarly it is possible to evaluate the different contributions to the displacement speed (see eq. 15).

\section{Tunnel closure}

The analysis was carried out on small subsets of the entire Da+ dataset varying in size from 30 points cubed to 80 points cubed, selected from the dataset based on a high density of occurrence of tunnel closure events. Each subset was scanned to find all the critical points, and each critical point was classified according to the analysis of Griffiths et al. [5]. Critical points with the tunnel closure topology were selected for further analysis.

Figure 3 shows one such sub-domain (left) and a slice taken through a plane in the middle (right), showing a clear tunnel closure event leading to flame pinch-off and the formation of a reactant pocket. For several such tunnel closure events, the dissipation and kinematic restoration terms were evaluated at DNS mesh points along the mesh line nearest to the critical point. This procedure avoids the need for interpolation, and allows all quantities to be evaluated directly from the DNS data. Both terms were then compared with the theory from section 2 . It should be noted that the theory is valid only close to each critical point, and that the spatial extent of its validity is not known a priori.

Each tunnel closure event was found to have its own and possibly unique large-scale geometry. Four different examples are provided in Fig. 4, which shows two-dimensional slices taken close to the critical point (left side) to- 

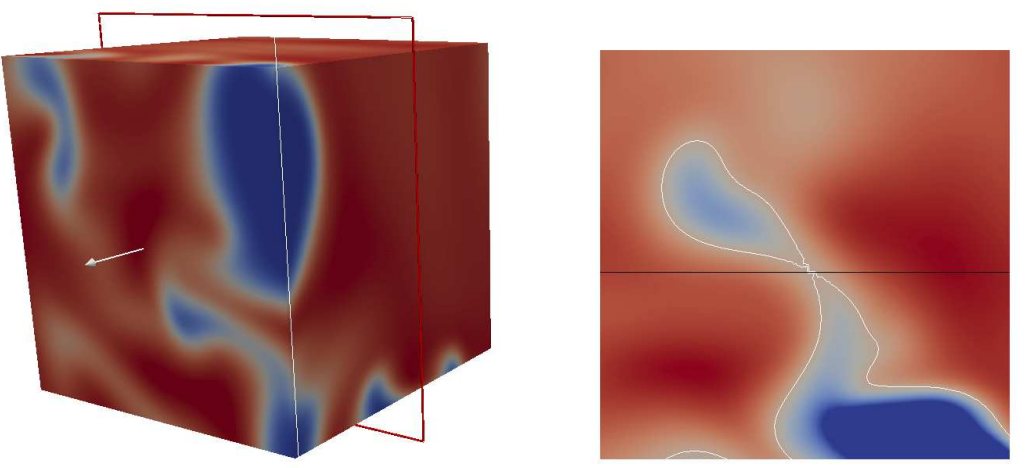

Figure 3: Example of a tunnel closure event. Colour scale runs from blue in reactants to red in products.

gether with corresponding plots of the dissipation and kinematic restoration terms (right side).

Figure 4a shows the same slice as in Fig 3b. The white contour on each slice represents a progress variable isosurface that corresponds as closely as possible to the tunnel closure event captured on this plane, while the straight black line indicates an axis that passes close to the tunnel closure point. Along this axis, the dissipation term (eq. 9) and the kinematic restoration term (eq. 11) were evaluated.

It is clear from the plots in the right column of Fig.4 that both the dissipation term and the kinematic restoration term reach their highest magnitudes in the vicinity of a critical point. Furthermore, the terms form a rough mirror image, having similar magnitudes but opposite signs, thus tending to cancel one another. This result is in good agreement with the theory and also with the two-dimensional DNS results of Kollmann and Chen [4]. Indeed the magnitudes of each term are similar to those found previously. 
The lack of complete cancellation between the dissipation and kinematic restoration terms is an indication that the axis of evaluation does not pass exactly through the location of the critical point. This is to be expected, since in general the critical point location will not lie on a DNS mesh line. Instead, the analysis method identifies the nearest DNS mesh line as the axis of evaluation, and therefore does guarantee that the critical point lies within a minimum distance corresponding to one-half of the DNS mesh spacing. Interpolation within a DNS mesh cell could be used to reduce the minimum distance and may well improve the degree of cancellation. Nevertheless, even in the present work, the axis of evaluation appears to be sufficiently close to the critical point for the theory to hold.

Figure $4 \mathrm{~b}$ shows a different subdomain containing a different tunnel closure event. Again, the same terms were evaluated along a line passing through the approximate location of the critical point. In this case it can be seen that there are two locations of interest on the same line, Again, both terms have large magnitudes close to each critical point and again the terms tend to cancel each other at both locations.

Figure $4 \mathrm{c}$ and $4 \mathrm{~d}$ show further subdomains containing different tunnel closure events, with planar views taken in different coordinate orientations. The terms are evaluated along the $y$-coordinate in Fig.4c and along the $z$ coordinate in Fig.4d. Again the results are consistent with the theory showing high magnitudes close to the critical point and a tendency to cancel each other.

It would be desirable to carry out a more complete statistical survey of the available critical points in order to confirm the generality of the results. 
4a)
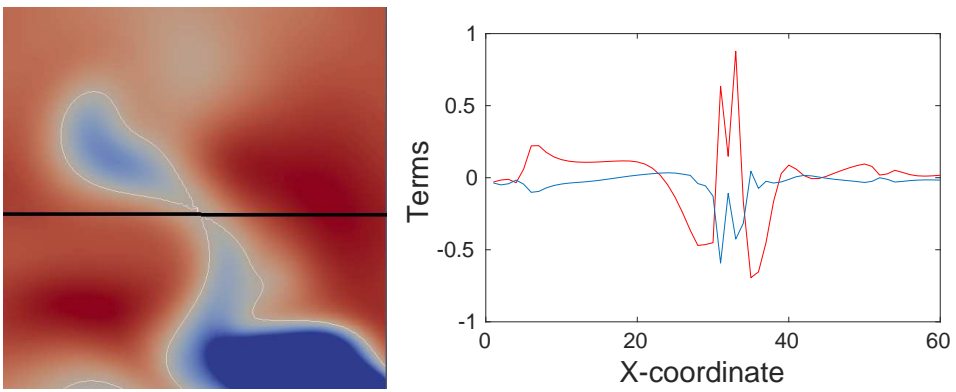

4b)
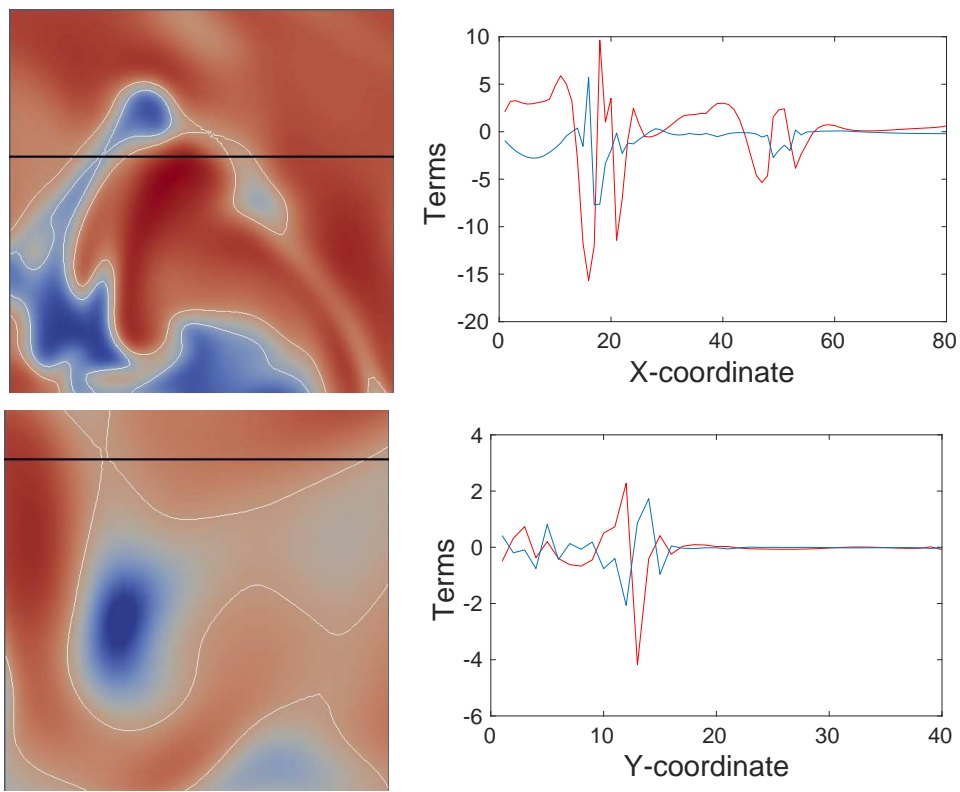

4c)
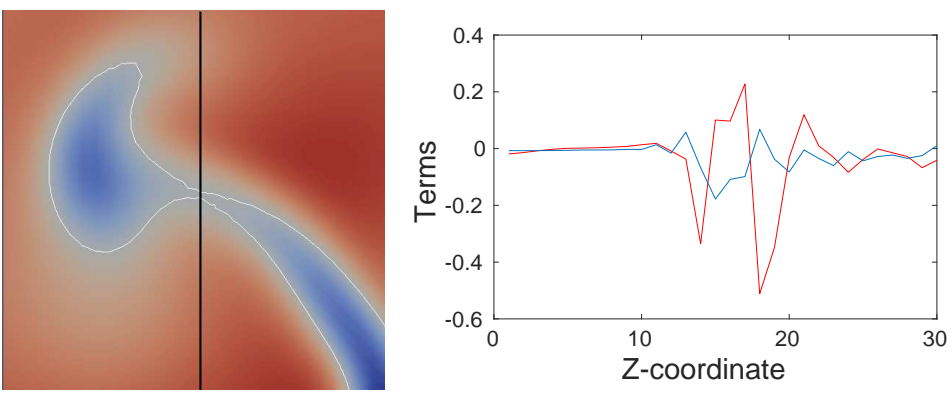

Figure 4: Examples of tunnel closure events (left column) together with (right column) the corresponding dissipation terms (in blue) and kinematic restoration terms (in red). 
This would be a major undertaking due to the large number of tunnel closure events, as well as the variety of scales, orientations and detailed geometrical features that are observed. Development of an automated procedure for this purpose is left to future work.

\section{Reactant pocket burnout}

In order to investigate the behaviour of displacement speed close to reactant pocket burnout, another subdomain was selected containing a fully formed reactant pocket. The displacement speed is evaluated on isosurfaces of progress variable in the vicinity of the critical point in the centre of the pocket. Linear interpolation in each coordinate direction is used to determine the location of the isosurface, and the corresponding value of displacement speed. Figure 5 (top) shows a slice through a reactant pocket burnout event with a set of progress variable isosurfaces (white contours) surrounding the critical point during a reactant pocket burnout event.

Figure 5 (bottom) shows the displacement speed $S_{d}$ plotted against progress variable. Here the values of $S_{d}$ have been averaged over each progress variable isosurface. It is clear that $S_{d}$ takes large values close to the critical point and falls sharply with increasing progress variable. This is consistent with the behaviour expected from theory, as expressed by eq. 15 .

\section{Conclusion}

Flame pinch-off leading to the formation of pockets has been investigated using both theory and DNS data. Previous work on topological analysis of the flame surface has identified four frequently-occurring configurations which are relevant to the pinch-off process. Using these configurations, a 

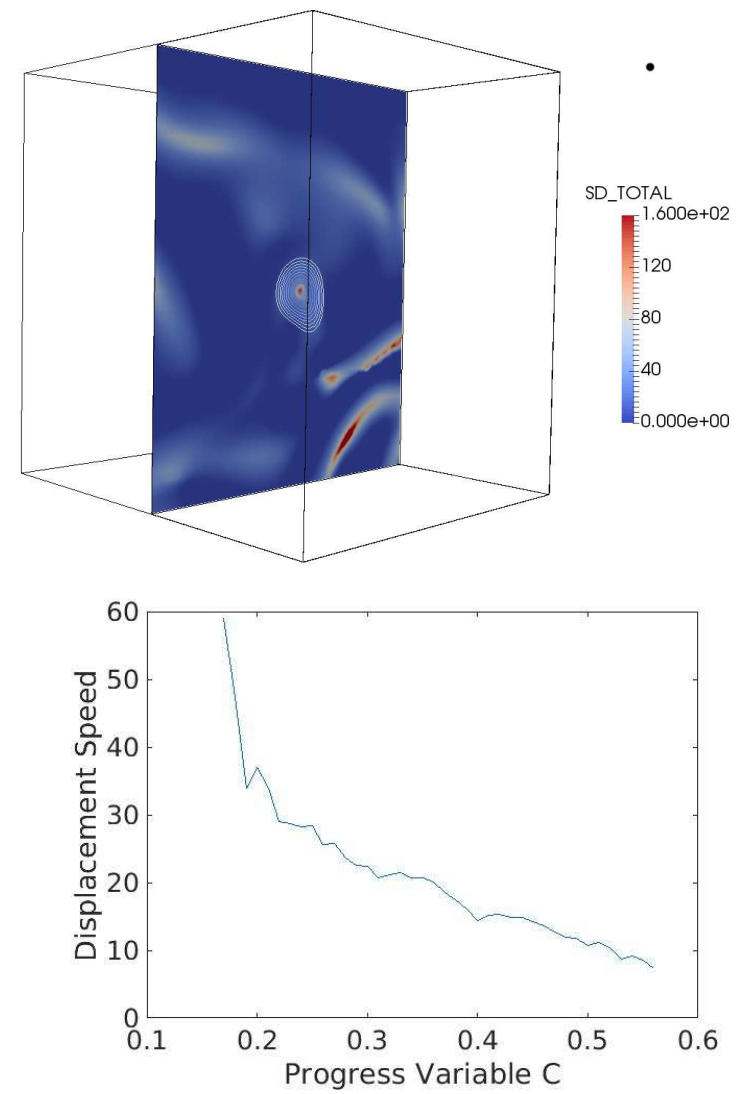

Figure 5: (Top) progress variable isosurfaces close to a critical point during reactant pocket burnout. (Bottom) Variation of displacement speed $S_{d}$ close to reactant pocket burnout previous mathematical analysis of the balance equation for SDF carried out in two dimensions using Morse theory has been extended to three dimensions. The main conclusions of the previous two-dimensional study are shown to be valid also in three dimensions, despite the greatly increased topological richness introduced by the extra dimension. The new analysis indicates that flame pinch-off occurs by means of a tunnel closure event leading to the formation of a reactant pocket. Conversely, a tunnel formation event may lead to the creation of a product pocket. 
Moreover, singular behaviour identified in the previous two-dimensional analysis is shown to occur also in three dimensions for both tunnel closure and tunnel formation events. Again the correspondence between two-dimensional and three-dimensional results is remarkable. Singularities arise at a critical point during pinch-off in the dissipation and kinematic restoration terms, and are shown to cancel each other out. A singularity is also shown to occur in the displacement speed at the critical point during the final burnout of a reactant pocket.

The predictions of the theory have been tested by comparison with a well-established DNS dataset for hydrogen-air flames. Several tunnel closure and reactant pocket burnout events have been identified and extracted from the dataset, and the relevant terms have been evaluated. The results indicate that large magnitudes of the dissipation and kinematic restoration terms are indeed observed close to the critical point during tunnel closure, and that the terms tend to cancel one another, in agreement with the theory. Large values of displacement speed are also observed close to the critical point during reactant pocket burnout, again in agreement with the theory.

Future work will focus on improving the DNS postprocessing techniques to capture the critical points more accurately in space and time, and to collect more data on the local scalar and velocity fields. An automated procedure will be required to handle the large number and variety of critical points. The implications of the observed behaviour for the modelling of flame propagation with rapid area change will be considered in greater detail. 


\section{Acknowledgments}

The work at Sandia National Laboratories was supported by the Division of Chemical Sciences, Geosciences and Biosciences, the Office of Basic Energy Sciences, the US DOE. Sandia National Laboratories is a multimission laboratory managed and operated by National Technology and Engineering Solutions of Sandia, LLC., a wholly owned subsidiary of Honeywell International, Inc., for the U.S. Department of Energy's National Nuclear Security Administration under contract DE-NA-0003525.

\section{References}

[1] R.S. Cant, S.B. Pope, K.N.C. Bray, Proc. Combust. Inst. 23 (1990) 809-815.

[2] N. Peters, Turbulent Combustion, Cambridge University Press, Cambridge, UK, 2000.

[3] W. Kollmann, J.H. Chen, Proc. Combust. Inst. 25 (1994) 1091-1098.

[4] W. Kollmann, J.H. Chen: Proc. Combust. Inst. 27 (1998) 927-934.

[5] R.A.C. Griffiths, J.H. Chen, H. Kolla, R.S. Cant, W. Kollmann: Proc. Combust. Inst. 35 (2015) 1341-1348.

[6] J.H. Chen, T. Echekki, W. Kollmann, Combust. Flame. 116 (1999) 1548.

[7] J. Milnor, Morse Theory, Princeton University Press, Princeton, NJ, 1963. 
[8] E.R. Hawkes, O. Chatakonda, H. Kolla, A.R. Kerstein, J.H. Chen: Combust. Flame 159 (2012) 2690-2703.

[9] J. Li, Z. Zhao, A. Kazarov, F.L. Dryer: Int. J. Chem. Kinet. 36 (2004) 566-575.

[10] J.H. Chen, A. Choudhary, B. de Supinski, M. DeVries, E.R. Hawkes, S. Klasky, W.K. Liao, K.L. Ma, J. Mellor-Crummey, N. Podhorszki, R. Sankaran, S. Shende, C.S. Yoo, Comput. Sci. Discovery 2 (2009) 015001. 